\title{
Impairments of Reaching Movements in Patients Without Proprioception. II. Effects of Visual Information on Accuracy
}

\author{
CLAUDE GHEZ, JAMES GORDON, AND MARIA FELICE GHILARDI \\ Center for Neurobiology and Behavior, New York State Psychiatric Institute and Program in Physical Therapy, \\ Columbia University, College of Physicians and Surgeons, New York, New York 10032; and INB-Consiglio Nazionale \\ delle Ricerche, Milan, Italy
}

\section{SUMMARY AND CONCLUSIONS}

1. The aim of this study was to determine how vision of a cursor indicating hand position on a computer screen or vision of the limb itself improves the accuracy of reaching movements in patients deprived of limb proprioception duc to large-fiber sensory neuropathy. In particular, we wished to ascertain the contribution of such information to improved planning rather than to feedback corrections. We analyzed spatial errors and hand trajectories of reaching movements made by subjects moving a hand-held cursor on a digitizing tablet while viewing targets displayed on a computer screen. The errors made when movements were performed without vision of their arm or of a screen cursor were compared with errors made when this information was available concurrently or prior to movement.

2. Both monitoring the screen cursor and seeing their limb in peripheral vision during movement improved the accuracy of the patients' movements. Improvements produced by seeing the cursor during movement are attributable simply to feedback corrections. However, because the target was not present in the actual workspace, improvements associated with vision of the limb must involve more complex corrective mechanisms.

3. Significant improvements in performance also occurred in trials without vision that were performed after viewing the limb at rest or during movements. In particular, prior vision of the limb in motion improved the ability of patients to vary the duration of movements in different directions so as to compensate for the inertial anisotropy of the limb. In addition, there were significant reductions in directional errors, path curvature, and late secondary movements. Comparable improvements in extent, direction, and curvature were produced when subjects could see the screen cursor during alternate movements to targets in different directions.

4. The effects of viewing the limb were transient and decayed during a period of minutes once vision of the limb was no longer available.

5 . It is proposed that the improvements in performance produced after vision of the limb were mediated by the visual updating of internal models of the limb. Vision of the limb at rest may provide configuration information while vision of the limb in motion provides additional dynamic information. Vision of the cursor and the resulting ability to correct ongoing movements, however, is considered primarily to provide information about the dynamic properties of the limb and its response to neural commands.

\section{INTRODUCTION}

In patients with sensory neuropathy, both reaching accuracy and postural control become degraded severely in the dark or when patients cannot see their limbs (Adams and
Victor 1981). This degradation when vision is absent generally has been attributed to the patient's inability to detect and consequently to correct errors. Similarly, in tracking tasks, they are unable to the maintain steady alignment of a cursor with a target when the cursor is blanked (Rothwell et al. 1982; Sanes et al. 1985). On the other hand, rapid single-joint movements, which depend mainly on feedforward mechanisms, may be performed relatively normally, albeit with increased variability. Systematic errors do occur, however, if the load opposing movement differs from what the patient expects (Rothwell et al. 1982; Sanes et al. 1985). Such observations underscore the importance of proprioceptive feedback for correcting errors in movement and posture (Matthews 1972; Nichols and Houk 1976).

In addition to its role in feedback, proprioceptive input also may contribute to accuracy by improving the planning of movement. However, the role of proprioception in the feedforward control of movement remains largely undefined. In an earlier study, we reported that patients with largefiber sensory neuropathy showed increased variability in the timing of agonist and antagonist contractions necessary to produce rapid isometric force impulses (Gordon et al. 1987). In carrying out those experiments, however, we found that by viewing their limb, patients were better able to select the patterns of muscle activation appropriate to generate force in a desired direction. In that study, subjects faced an oscilloscope screen, where a target was displayed together with a cursor whose position varied with elbow force. Their arm was abducted and outside their field of view. Although control subjects quickly learned to produce flexor or extensor force pulses to match changes in target position on the screen, deafferented patients frequently made wrong-direction responses, flexing when they should have extended, or vice versa. [It is interesting to note that wrong-direction responses also are evident in the illustration of 3 and $9^{\circ}$ movements performed without vision by deafferent patients in a study by Sanes and colleagues (1985)]. It was only when their arm was repositioned so that it and the oscilloscope screen could be seen concurrently that the patients were able to avoid making such errors. Vision of the limb apparently was needed for the patients to correctly specify which muscles to contract. This raised the possibility that the difficulty experienced by deafferented patients in the dark might reflect a distorted or impoverished internal representation of their limb. Thus apart from its role in providing 
error information used for feedback corrections, proprioceptive input might be critical for the programming process itself. These observations in an isometric task are what led us to undertake the current series of experiments.

The main purpose of the present study was to determine whether, in patients without proprioception, vision of the limb itself might improve the planning of subsequent movements made without vision. In addition, we wished to determine the nature of the visual information that might be useful as well as the nature of the trajectory improvements that might occur. As in the preceding paper (Gordon et al. 1995), subjects moved their hand on a digitizing tablet from a starting position to a target. Starting and target positions were displayed on a computer monitor together with a screen cursor representing the hand position. We now examined the effects of allowing subjects to view the screen cursor or their arm and hand in the workspace during or prior to movement. By indicating the distance from current hand position to the target, vision of the cursor on the screen provides feedback information which could, within the limits of reaction times and movement times, be used to correct errors. Because the target was not displayed on the tablet, vision of the limb in the workspace does not provide feedback information per se. When available before movement, this information can only be used to provide information about limb configuration or other information that might be needed to program movements accurately.

In the preceding paper (Gordon et al. 1995), we showed that, in reaching toward visual targets, patients with largefiber sensory neuropathy make large errors in direction and extent, and their hand paths are abnormally curved. These errors were evident very early in movement trajectories, suggesting an impairment in feedforward control. In addition, the ability of patients to compensate for directional variations in the inertial resistance to movement by varying movement time was impaired substantially, leading to systematic errors. Therefore, we now wished to determine whether visual information about the limb can substitute for proprioceptive input in compensating for inertial variations in initial acceleration.

Some of these results have been presented previously in abstracts (Ghez et al. 1988; Gordon et al. 1987, 1990) and a symposium (Ghez et al. 1990).

\section{METHODS}

Subjects were eight controls with intact sensation and no known neurological deficits and three patients with severe sensory neuropathy. Different control subjects participated in different sets of experiments. The patients were the same as those reported in the previous study $(M A, G L$, and $C F)$ and are described in greater detail there and elsewhere (Forget and Lamarre 1987 for $G L$; Sainburg et al. 1993 for $M A$ and $C F)$. Patient $M A$ was studied most extensively for several years. Comparable results are reported for the two other patients, who were studied for a lesser period of time ( $G L$ and $C F$ were studied in morning and afternoon sessions for 3 and 4 days each, respectively).

The experimental methods used in this study were similar to those described in the companion paper (Gordon et al. 1995) and elsewhere (Gordon et al. 1994a,b). Briefly, subjects were seated facing a computer monitor and moved a hand-held cursor on a digitizing tablet while viewing the screen of a computer monitor.
The subjects were to first position a screen cursor (which reflected the position of the hand cursor on the tablet) by moving their hand to a starting position, displayed as one of two small circles on the monitor. Then, upon presentation of a tone, subjects were to move their hand in a single quick uncorrected movement to a target location indicated by the second circle on the screen. In the standard, or baseline condition (No Vision), the cursor disappeared upon presentation of the tone though the target continued to be displayed. Knowledge of results (KR) was, however, provided in the form of a hand path displayed on the computer screen at the end of each trial. Vision of the arm was prevented by the combination of a two-way mirror over the tablet and a drape covering the upper arm and shoulder.

In most experiments, the tablet was at waist level so that the upper arm was close to vertical at the initial position of the various movements studied. In some experiments, to eliminate the effect of gravity and simplify the mechanical forces acting on the limb, the tablet was placed at shoulder level and the arm was supported in the horizontal plane (Gordon et al. 1994a).

Subjects were tested in blocks of 18-48 trials in which targets in different locations were presented in a pseudorandom sequence. In the course of these experiments, we used two different target arrays. In early experiments, whose results are illustrated in Fig. 1 , we used targets at three distances $(7.5,15$, and $22.5 \mathrm{~cm})$ from each of three starting positions located near one another and close to the subject's body. The three targets were, however, in different directions $\left(30,90\right.$, and $\left.150^{\circ}\right)$ for each of the initial positions. In later experiments, 12 or 24 targets were positioned radially at regular intervals and a constant distance $(7.5 \mathrm{~cm}$.) from a common starting position located $25-30 \mathrm{~cm}$ directly in front of the body.

To assess how visual information influenced trajectory formation, we compared performance in the standard No Vision condition with four other conditions in which different information was provided. We examined the effects of visual feedback during movement by displaying the screen cursor during movement (Cursor Feedback condition). We began our studies of the effects of viewing their hand and arm by allowing patients to see their limb between trials (Prior Arm Vision condition). To allow vision of the arm, a light under the two-way mirror was turned on and the drape covering the subjects shoulder was removed for a few seconds. In most experiments, this was done while the patients maintained their hand in a stationary position at the starting position, immediately before movement. However, in a later phase of the studies (in patients $M A$ and $C F$ ), to provide the patients with additional dynamic information, we allowed them to see their upper arm, forearm, and hand during movement itself. For this purpose we turned on the light and removed the drape on alternate trials in the block (Alternate Arm Vision condition). During trials with vision of the limb, subjects generally maintained their gaze on the screen, seeing their arm only in peripheral vision or looked at it only intermittently. We assessed the effects of cursor feedback on the screen on the planning of later movements by displaying the cursor during movement on alternate trials (Alternate Feedback condition). The rationale for this was that feedback during the movement provides information about the limb's response to immediately preceding motor commands that might be used to plan future movements in other directions. Performance under these conditions were always compared with No-Vision data obtained in blocks that were run in the same testing session.

The procedures used for data analysis are described in previous papers (Gordon et al. 1994a,b). Briefly, movement paths were smoothed using a cubic spline, and tangential velocities and accelerations were computed using standard methods. Movement onsets, peak accelerations, peak velocities, and terminations were determined for each movement using automatic computer routines and checked and corrected when necessary. 
As noted in the preceding paper, patients frequently made secondary movements after the main transport phase, which we will refer to as the primary movement. These were unintentional and unnoticed by the patients. Therefore, for each movement we marked two end points. The end of the primary movement (End1) was taken as the zero-cross or minimum of the tangential velocity (this typically occurred at a point of relatively large curvature in the hand path). The end of the secondary movement (End2) was marked either when the hand had come to a complete stop for $\geq 0.5 \mathrm{~s}$ or when data collection was discontinued. The latter only occurred when the hand was continuing to drift very slowly or when it came to the edge of the tablet (see RESULTS section below, regarding secondary movements, and Fig. 6 for more details). The straight-line distance from End1 to End 2 was used as a measure of the extent of the secondary movements.

The distance from the primary movement end point (End1) to the target, termed here movement error, was used as a global measure of accuracy. We also computed extent and direction errors separately. Movement extent was defined as the length of a straight line from the starting point to Endl. Movement direction was computed as the orientation of this vector. Extent error was computed as the difference between the actual movement extent and the distance to the intended target (i.e., the distance of the target from the initial hand position). Directional error was defined as the difference between the target direction and the direction of the vector at End1. In our convention, a directional error was called negative when actual movement direction was clockwise relative to target direction and positive when it was counterclockwise. To quantify the curvature of each trajectory, we computed, for each trial, the absolute value of the difference between the movement direction at the end point and at the peak acceleration. For both extent and directional errors, we computed two global masures of error: mean absolute error and mean variable error. Mean absolute error is the mean of the absolute values of the errors in direction or extent on each trial. Mean variable error is the mean of the absolute values of the difference between each trial's direction or extent and the mean direction or extent for all the trials to a specific target. Variable error therefore excludes contributions of constant errors and of systematic errors that vary with the direction of movement.

The relationship between movement direction and various trajectory parameters in tasks requiring movements in 24 or 12 directions was characterized by means of a nonparametric curve-fitting procedure, called LOWESS, or locally weighted scatterplot smoother (Cleveland 1979).

\section{RESULTS}

\section{Screen cursor feedback and prior limb vision reduce trajectory errors in deafferented patients}

The effects of vision of the cursor on the screen and of vision of the hand and arm on the tablet before movement are illustrated in Fig. 1, which shows the hand paths and end-point distributions of movements made by patient $G L$ to three targets in one of three directions tested. In the No Vision trials, in which the cursor disappeared when the go signal was presented, large errors in direction and extent are evident (Fig. 1, top). In trials with Cursor Feedback, in which the cursor remained visible on the screen during the entire trial, the hand paths are clustered together more tightly and end point distributions are smaller (Fig. 1, middle). In Prior Arm Vision trials, the arm and hand were uncovered transiently for a few seconds before each movement. This

\section{Patient GL}

A Movement Paths

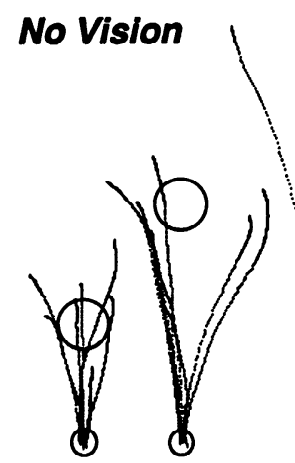

Cursor Feedback
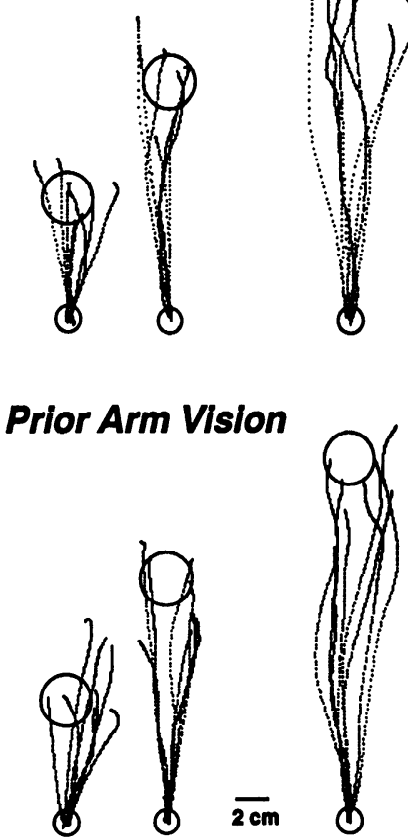
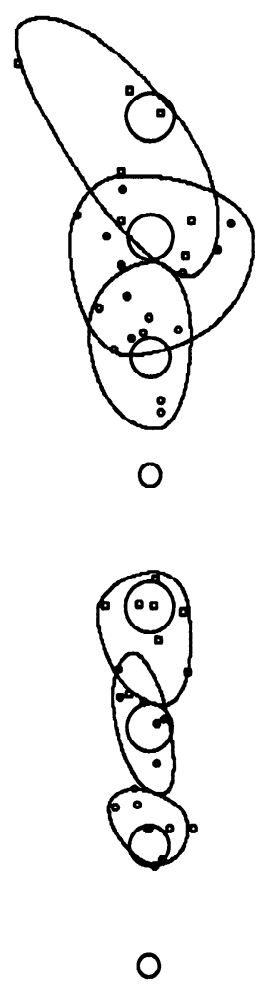

O

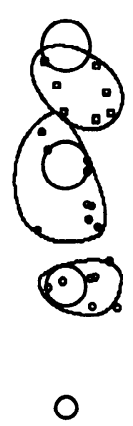

\section{B End Points}

FIG. 1. Effects of different types of visual information on accuracy of reaching movements in patient GL. Movement paths $(A)$ and end points $(B)$ are shown for 6 movements to each of 3 targets at different distances in $90^{\circ}$ direction. Small circles are the starting positions and large circles the targets. $A$ : movement paths plotted as dots representing the position of the hand at successive 20 -ms intervals. $B$ : movement end points (end 1 ) for paths shown in $A$. End points to near target, $\bigcirc$; end points to middle target, $\bullet$; and end points to the far target $\square$. Each set of end points is fitted with a contour that approximates the shape of the distribution. The orientation of the contour was computed by the method of principal components and its size and shape was based on the interquartile range in each of the major axes (Gordon et al. 1995). See text for explanation of conditions.

also produced a reduction in path and end point dispersion (Fig. 1, bottom), similar to the reduction that occurred with cursor feedback. In both conditions (Cursor Feedback and Prior Arm Vision), the increased accuracy resulted from a combination of reduced directional variability and reduced extent variability. As noted in the preceding study, for the 
same target set and the same number of trials, three control subjects performing in the no-vision condition showed greater accuracy than the patients. However, whereas cursor feedback produced modest but nonsignificant improvements in accuracy in control subjects, vision of the limb before movement did not.

Although the effect of screen feedback was roughly the same for all three patients, the effect of prior vision of the limb varied. In one patient, $M A$, the effect was comparable with those illustrated in Fig. 1 and was present for all target directions. In $G L$, the effect was only consistently present for the direction illustrated, and in $C F$, prior vision of the arm at rest between trials was without effect. Whereas the effects of cursor feedback can be attributed, at least in part, to corrections during movement, this cannot be the explanation for the reduction in errors with prior vision of the limb. Rather, prior vision must have increased accuracy by improving the planning of movement.

\section{Prior vision of the moving arm reduces both systematic and variable errors}

As noted in the preceding paper, the inaccuracy of deafferented patients results both from general increases in trajectory variability and from specific errors that vary systematically with the direction of movement. In particular, we found large direction-dependent errors in movement extent that reflected the anisotropy in the limb's inertial field. Thus whereas direction-dependent variations in peak acceleration were comparable in controls and patients, only in controls was this anisotropy substantially compensated by directiondependent variations in movement time. We therefore suggested that an important determinant of inaccuracy in deafferentation was a failure to adapt motor commands to the biomechanical characteristics of the limb. In the present study, therefore, we sought to determine if vision of the limb might enable patients to improve their compensation for variations in limb inertia.

To maximize the amount of information about limb inertia that might be provided by prior vision, we now allowed subjects to view their limb while it was in motion. This was done by presenting blocks of trials in which, on alternate trials, subjects were either allowed to see their limb or prevented from doing so. The same target was never presented on successive trials. We reasoned that vision of the limb's response to prior motor commands might provide subjects with dynamic information about its properties that they could use to refine the programming of subsequent movements. Indeed, in an initial experiment with patient $G L$, this appeared to produce a greater improvement in performance, relative to the No Vision condition, than simple vision of the limb at rest before movement. However, analyses carried out when the patient was no longer available for study revealed that the movement directions sampled in the two conditions were not the same and therefore the errors could not properly be compared. In subsequent experiments, done with patients $M A$ and $C F$, we used targets in 12 or 24 equally spaced directions $7.5 \mathrm{~cm}$ from a common initial hand position. Hand paths and errors made with and without vision of the limb were separated for analysis and compared with those of movements made in No Vision trials presented immediately before or after.

Figure 2 shows the effects on hand trajectories of alternating trials with and without vision of the limb in patient MA. As noted in the previous paper, there are large directiondependent errors in movement extent in the No Vision condition, such that movements along the $30-210^{\circ}$ axis are markedly hypermetric (Fig. 2, top left). Moreover, for some directions, the hand paths are highly curved, and they are grouped into zones of concentration and sparseness. These abnormalities were reduced substantially in the movements when vision of the limb was provided on alternate trials. However, little difference is evident between the trials with (Alternate Vision) and without (Alternate No Vision) vision of the limb (Fig. 2, top right and middle).

To determine whether vision of the arm and hand before or during movement improved the patients' ability to compensate for variations in limb inertia, we first examined the directional variations in peak acceleration, movement extent and movement time in the different conditions (Fig. 2, bottom 3 rows). In the No Vision condition, the large directiondependent variations in peak acceleration (Fig. 2, left, top scatter plots) are paralleled by equally large variations in movement extent (left lower scatter plots) whereas movement time does not show a clear dependence on movement direction (middle row scatter plots). However, the directiondependent variation in movement extent is markedly reduced in movements with and without vision of the arm in the alternate vision condition (lower graphs in middle and right of Fig. 2). This reduction is associated with the emergence of a clear direction-dependent variation in movement time which now mirrors, as in control subjects, the variations in peak acceleration. It also should be noted that the variability around the fitted lines is markedly reduced in both the Alternate No Vision and Alternate Vision trials relative to those with No Vision.

As described in the preceding paper (Gordon et al. 1995 ), we estimated the degree to which movement extent and movement time depended on inertia by computing the correlations of movement extent and movement time with peak acceleration. Figure 3 shows the percentage of the variance $\left(r^{2}\right)$ in movement extent (Fig. $3 A$ ) and movement time (Fig. $3 B$ ), explained by variation in peak acceleration in the different conditions for patients $M A$ and $C F$. For purposes of comparison, the median $r^{2}$ values for seven control subjects performing without vision are shown as solid lines with an empty circle. In both patients, movement extent was highly correlated with peak acceleration when they performed without vision. Control subjects showed little or no correlation. Vision of the limb decreased this dependence and increased the variance in movement time explained by peak acceleration. Interestingly, this decrease was similar for movements in which vision was available during movement and for movements in which vision was available on the preceding trial. Thus vision of the limb, both concurrently and before movement, substantially improved the patients' ability to adapt their motor commands to the inertial properties of their limb.

To assess the effect of vision on accuracy itself, we 
No Vision
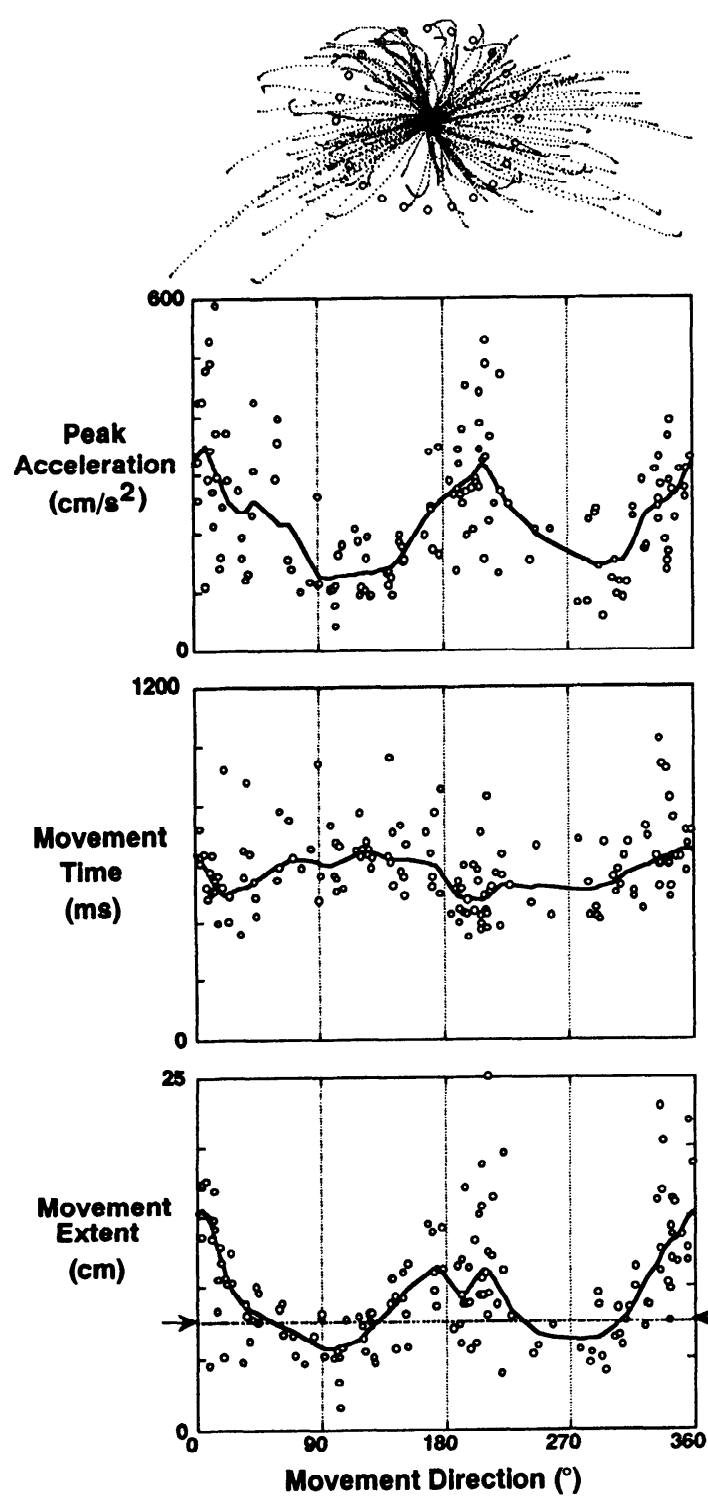

Alternate No Vision

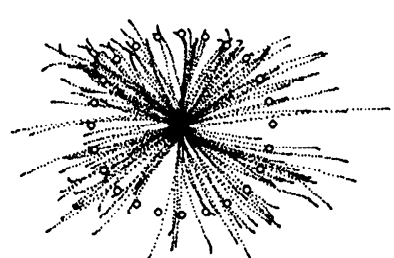

Alternate Arm Vision

$\overline{\mathrm{acm}}$

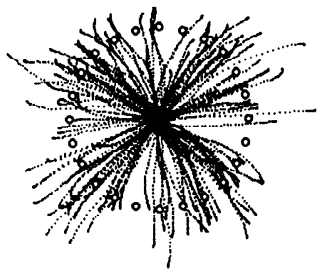

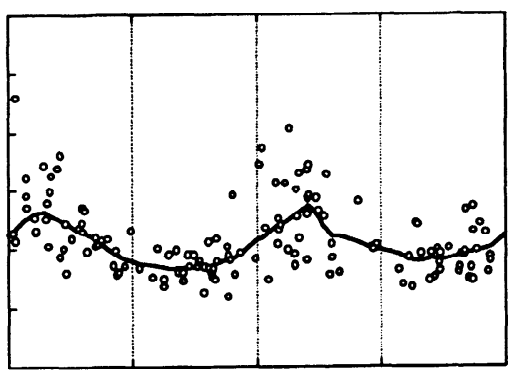
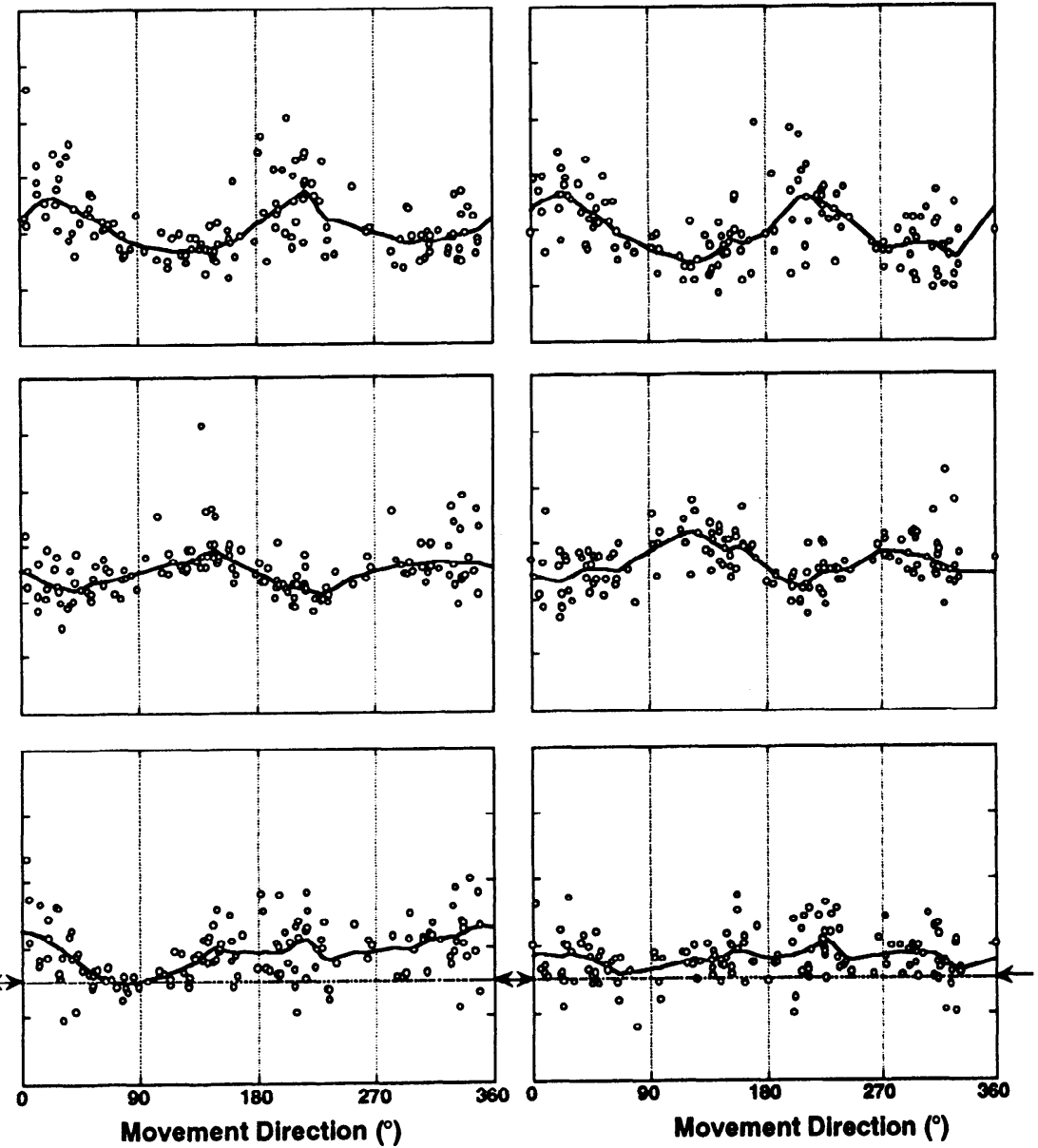

FIG. 2. Effect of vision of the limb during movement in patient MA. Movement paths and trajectory variables are shown for 144 movements in 24 directions for each condition. Left: No Vision. Middle and right columns: vision of the arm on alternate trials. Middle column: Alternate No Vision, alternate movements performed without vision. Right column: Alternate Vision, alterante movements performed with vision. In the 3 bottom rows, the solid lines through the data points represent a best-fitting line computed using locally weighted scatterplot smoother (LOWESS, see METHODS). In the bottom row, the dashed line indicates the target distance.

compared the mean absolute errors in extent and direction, (Fig. 4, open bars), the mean variable errors (Fig. 4, shaded bars), and the mean curvature in the three conditions using analysis of variance, followed by a post hoc test (Fisher's Protected Least Significant Difference [PLSD]). Small stars over the bars in the two alternate conditions indicate when the error is significantly less (Fisher's PLSD: $P<0.05$ ) than in the No Vision trials. In both patients, vision of the limb during movement produced the largest reductions in all these errors. Vision of the limb during the preceding trial also reduced the errors, but the degree of reduction was consistently less. In $p a$ - tient $C F$, differences in errors between the Alternate No Vision trials and the No Vision trials are significant except for the absolute extent errors. The reason the mean absolute extent errors remained high in the alternate No Vision trials for this patient, despite the large drop in the dependence of extent on initial acceleration, was the development of systematic overshoot errors for all target directions. In three control subjects in the No Vision condition, the mean absolute and variable errors as well as curvatures were much smaller than in either $M A$ or $C F$. Vision of the arm did not appreciably further reduce these errors (dashed lines in Fig. 4 histograms). 
A Movement Extent / Peak Acceleration

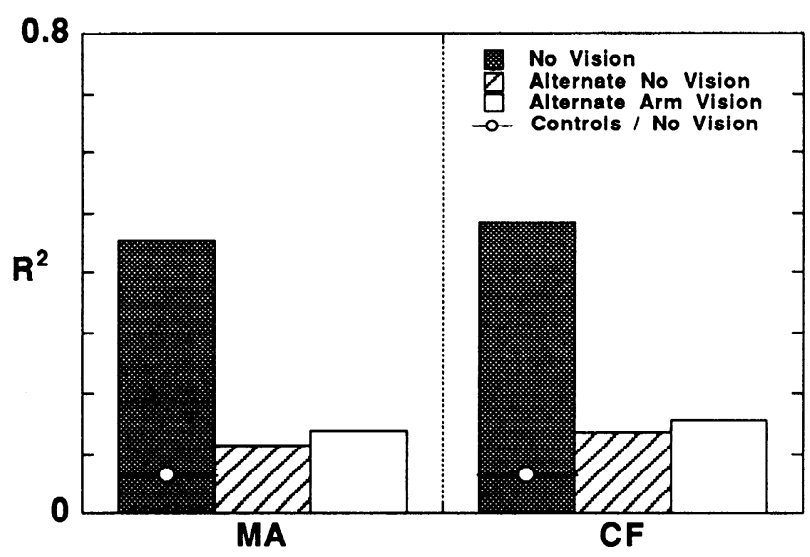

B Movement Time / Peak Acceleration

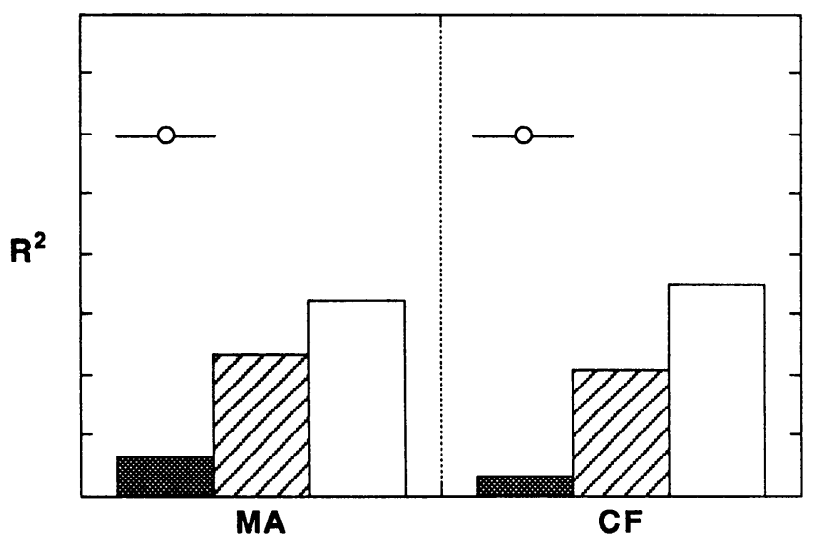

FIG. 3. Squared correlation coefficients $\left(r^{2}\right)$ between movement extent and peak acceleration $(A)$ and movement time $(B)$ in 2 patients $(M A$ and $C F$ ) performing in 3 conditions: No Vision (shaded bars), No Vision Alternate (hatched bars), and Full Vision (unfilled bars). In both plots, median $r^{2}$ values for 7 control subjects performing without vision are shown as lines with an empty circle.

\section{Screen cursor feedback improves the programming of subsequent movements}

We have seen that deafferented patients are able to reduce reaching errors through feedback by monitoring the position of the cursor on the screen during movement. However, vision of the cursor during movement also provides dynamic information about the limb's response to the subjects' neural commands. Therefore, we next asked whether patients could use this information to improve performance on a subsequent trial performed without cursor feedback. To address this question, we examined trajectory errors in trial blocks in which subjects had screen feedback, rather than vision of the arm, on alternate trials. Figure 5 shows the mean absolute and variable extent and direction errors as well as the mean curvature in the trials with and without screen feedback. These are compared with corresponding measures made in the No Vision condition performed immediately before. As in Fig. 4, stars over the bars for the alternate conditions indicate when these errors are significantly less than in the No vision condition (Fisher's PLSD: $P<0.05$ ). As expected, in both patients, visual feedback on the screen pro- duced large reductions in extent and direction errors. In patient MA similar changes were present on the subsequent trial when she could neither see the cursor nor her arm. In $C F$, prior vision of the cursor (like prior vision of the limb) significantly reduced extent variability and directional errors of subsequent trials, but mean extent errors were not reduced. Thus both recent vision of the arm and feedback of the cursor produced more substantial improvement in $M A$ than in $C F$. Two factors might plausibly have contributed to this intersubject difference: differences in the degree of practice in our task and the subject's age. At the time of testing, $M A$ had been studied for many more sessions and was almost 25 years younger than $C F$ at the time of her illness. Interestingly, in both subjects, mean curvature was higher in trials in which screen feedback was available than in the subsequent trials without feedback. Presumably, this reflected corrections of directional errors made during the course of individual movements. In three controls tested in these conditions, cursor feedback did not produce appreciable error reduction, and movements in the subsequent trials without vision or feedback also were unchanged compared to the $\mathrm{No}$ Vision trials (dashed lines in Fig. 5 histograms).

\section{Prior vision of the moving limb improves the specification of movement end points}

In the preceding paper, we noted that a characteristic feature of the movements made by patients without proprioceptive sensation is the presence of conspicuous secondary movements occurring after the initial velocity minimum (Gordon et al. 1995). These were typically slower and took the hand in a different direction than the direction of the main transport phase of the movement. We therefore concluded that the intended posture programmed for the end of the movement in patients might substantially differ from the spatial location of the point toward which the movement was initially aimed.

Because prior vision of the limb might reduce the disparities between aimed locations and intended postures, we next examined the changes in secondary movements in the alternate vision conditions. For this, we measured the lengths of the hand paths between the ends of the primary and secondary movements in no-vision and alternate arm vision blocks (i.e., the straight-line distance from End1 to End2). In these experiments, particular care was taken to avoid discontinuing data collection before the movement had come to a complete stop. In a few cases, the end of the secondary movement was marked prematurely, i.e., before the movement had come to a complete stop. This was necessary either because the hand had come to the edge of the digitizing tablet or because the hand was drifting at such a slow rate that its continued movement was not detected by the experimenter. Because this occurred primarily in the No Vision blocks, this measure slightly underestimates the true differences between conditions.

Figure $6 \mathrm{~A}$ shows only the secondary movements corresponding to the movement paths presented in Fig. 2. These were made by patient MA in the No Vision and the Alternate Vision conditions. In Fig. $6 B$, two sample hand paths and the corresponding tangential velocities are shown with the locations of 

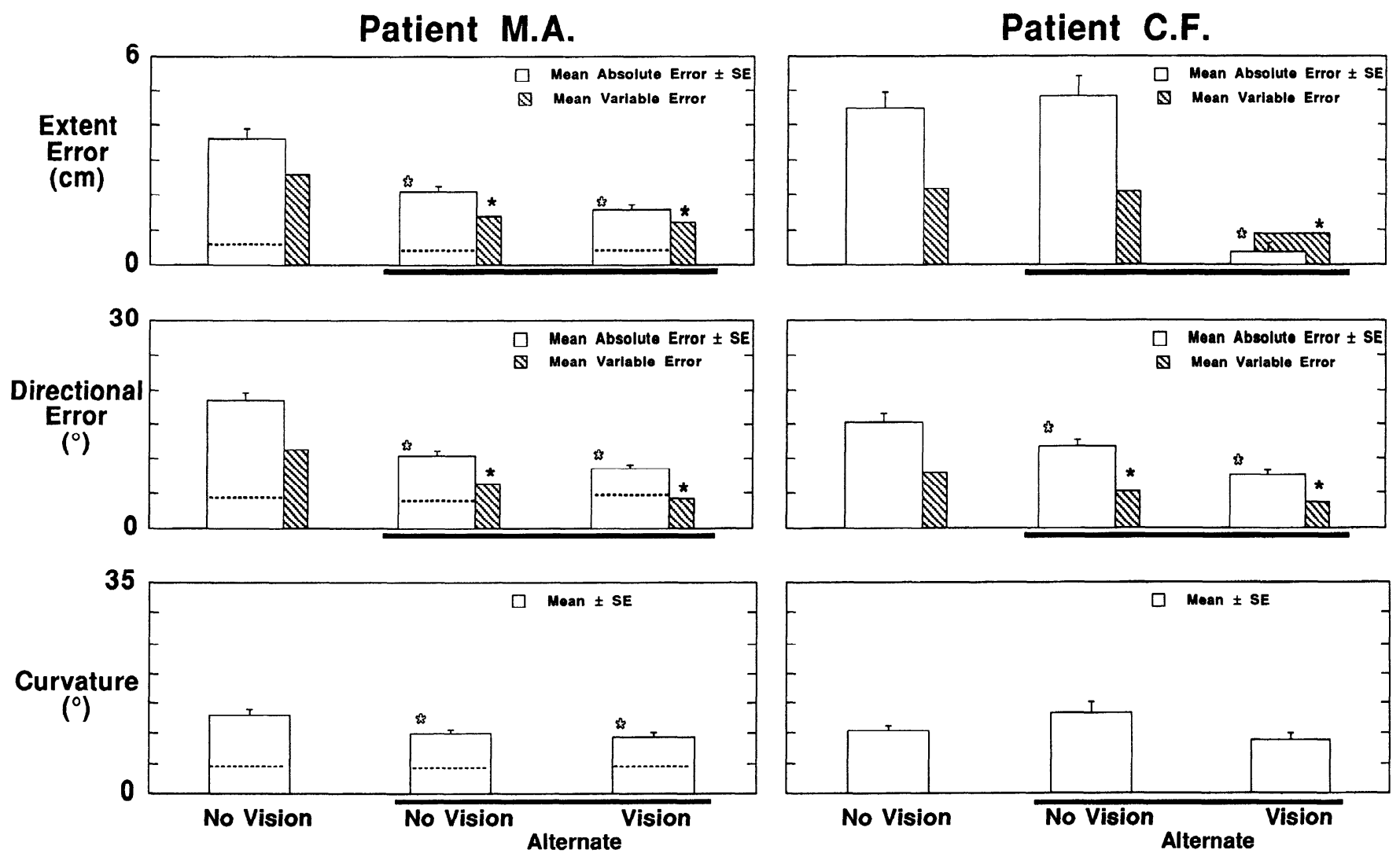

FIG. 4. Mean extent errors (top), directional errors (middle), and curvature (bottom) in 2 patients ( $M A$ and $C F$ ) performing in No Vision and Alternate Arm Vision conditions. For movement extent and direction, mean \pm SE (unfilled bars) and mean variable error (shaded bars) are shown. Analysis of variance (ANOVA) showed significant differences between movements performed in the 3 conditions $(P<0.001)$. When present, stars indicate that the value is significantly less than that in the No Vision condition (Fisher's PLSD, $P<0.05$ ). Dotted lines indicate mean errors for 3 normal subjects tested under the same condition.

first and second end points (End1 and End2) marked. The secondary movements are abolished almost completely in trials with vision of the limb, a finding that can in part be attributed to visual feedback. Although secondary movements are still present in the alternate trials without vision, they clearly are reduced substantially in comparison with those made in the No Vision block. Figure $6 C$ shows the mean secondary movement path lengths for both $M A$ and $C F$ for the same three conditions. In both patients, the path lengths are reduced significantly in the alternate vision blocks. It is interesting to note that whereas in $M A$ the secondary movements in alternate trials without vision had longer lengths than in those with vision, in $C F$ the lengths were virtually identical for both sets of trials. Note that the secondary movements often increase the absolute errors; therefore they cannot be simply corrections for errors of the primary movements. Thus in addition to improving the aiming of the primary movements, prior vision of the screen cursor during movement provides information that can be used to reduce the mismatch between the initial movement and the final posture.

\section{Improved accuracy produced by vision of the limb is transitory}

The beneficial effects of vision of the limb were transitory and decreased progressively once the patients could no longer see their limb. Figure 7 shows the time course of this decay in an experiment carried out with patient MA. In this experiment, there was an initial group of 24 trials in which the patient was given targets in the $150^{\circ}$ direction on the screen and made reaching movements with vision of her limb and KR available. Then, her hand and arm were covered and the series of trials continued while $\mathrm{KR}$ was provided as path displays after each movement for 36 trials during 15 min. The same procedure (vision of the arm and KR followed by no vision of the arm and KR) was repeated in a second block of trials. Figure $7 A$ shows representative paths (drawn as small circles at $20 \mathrm{~ms}$ intervals) of responses occurring in the indicated intervals after vision of the limb was blocked. Figure $7 \boldsymbol{B}$ shows the mean distance from the targets to the movement end points (termed here movement errors) and the extents of the secondary movements in successive time intervals for the two blocks of trials.

It can be seen from the sample hand paths that both endpoint errors and secondary movements rapidly increased in the first few trials performed without vision of the limb. The primary movements show increasing hypermetria and curvatures in the hand paths. The bar graphs show that these errors reach relatively stable values after $\sim 5 \mathrm{~min}$. Thus although the information provided by vision of the limb is retained and can be used to program movements when it is 

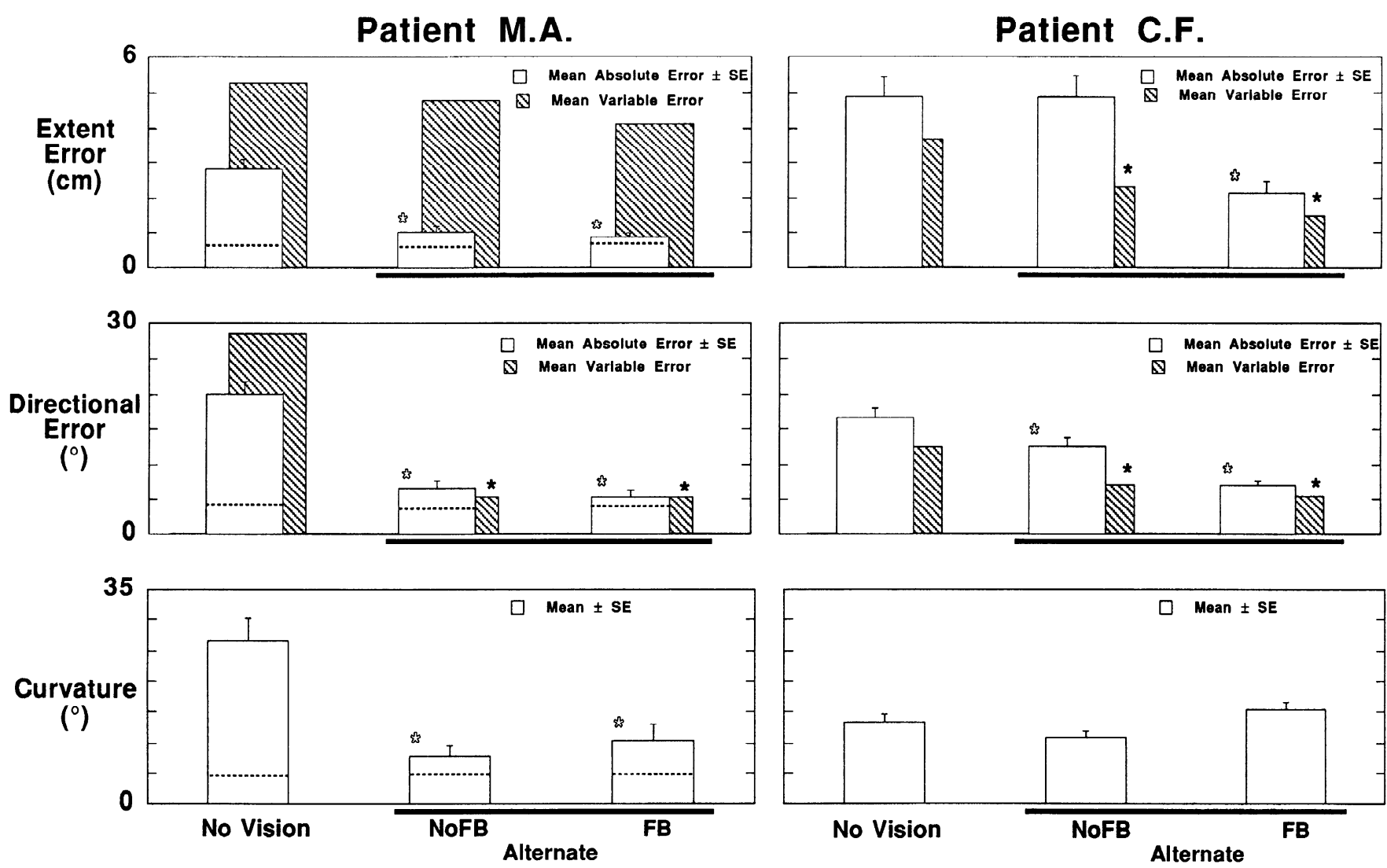

FIG. 5. Mean extent errors ( $t o p$ ), directional errors (middle), and curvature (bottom) in 2 patients ( $M A$ and $C F$ ) performing in No Vision and Alternate Feedback conditions. For movement extent and direction, mean \pm SE (unfilled bars) and mean variable error (shaded bars) are shown. ANOVA showed significant differences between the 3 conditions $(P<0.001)$. When present, stars over the bars for the 2 alternate conditions, indicate that the value is significantly less than that in the No Vision condition (Fisher's PLSD; $P<0.05$ ). The dotted lines indicate mean absolute errors for 3 normal subjects tested under the same conditions.

no longer available, the memory is evanescent and requires frequent updating.

\section{DISCUSSION}

The primary purpose of this study was to analyze how patients deprived of proprioception by large-fiber sensory neuropathy use visual information about the limb to improve their control of hand trajectories. There were three major findings. First, vision of the screen cursor or of the arm during the course of movement produces large reductions in all measures of error. This confirms that deafferented patients can use visual feedback to correct movement errors. Second, vision of the cursor on the screen or of the arm before movement also increases accuracy. A significant feature of the change in performance with prior vision is an improved ability to compensate for direction-dependent variations in limb inertia. Third, the gains produced by vision of the limb or of the screen cursor decay rapidly when visual information is no longer available. Thus accuracy in the aiming of movements to visual targets depends critically on short-term learning mechanisms.

\section{Effects of vision during movement}

Improvements in accuracy produced by vision of the cursor during movement are, at least in part, attributable to the correction of initial errors through feedback. Indeed, because the target remained visible on the screen and average movement times were $500-600 \mathrm{~ms}$, the patients, in principle, could evaluate its distance from the cursor to make adjustments to the movement while it was in progress. Vision of the limb during movement, on the other hand, does not provide explicit information about the distance of the hand to the target because the target was not displayed on the tablet. Moreover, even when the limb was visible during movement, both patients and controls tended to keep their gaze fixed on the screen and saw their limb primarily in peripheral vision. Thus whereas the improvement in accuracy might have been mediated by adjustments in the course of movement, additional contributions of feedforward mechanisms are likely also to have been important.

\section{Effects of prior vision}

Perhaps the most unexpected finding of this study was that vision of the limb during a given movement reduced trajectory errors in subsequent movements to targets in different directions. Therefore, vision of the limb provided the patients with information about properties of their limb that they could use to program a range of movements to different targets. We hypothesize that this information is 

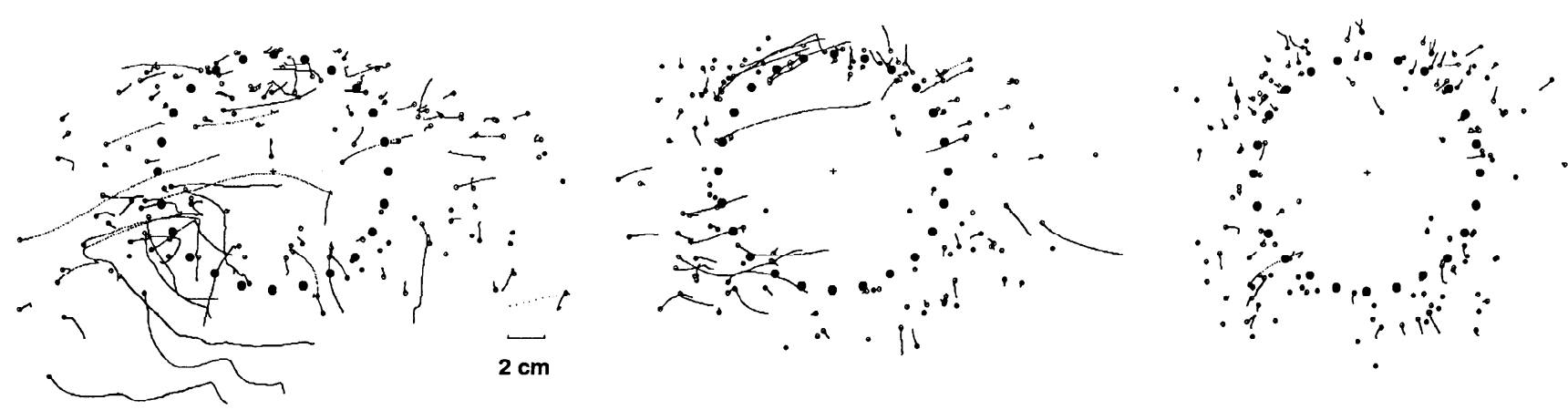

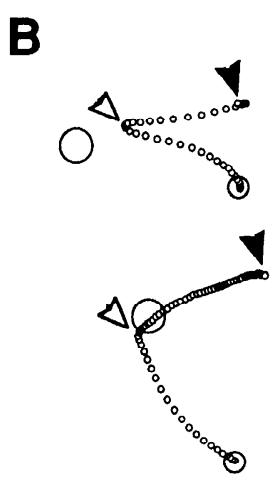

$2 \mathrm{~cm}$

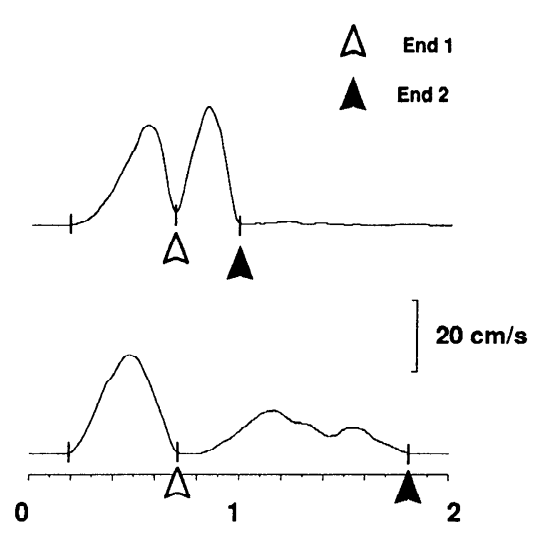

(s)

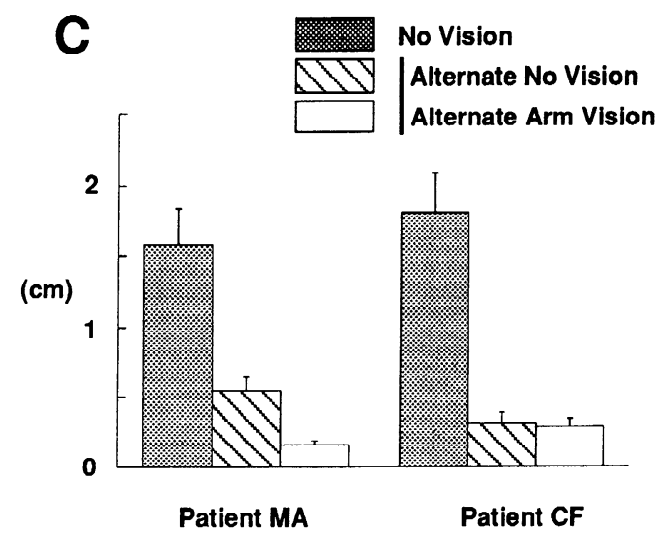

FIG. 6. Effect of vision during movement on secondary movements. $A$ : hand paths from Endl to End2 (ends of movements shown in Fig. 2) are drawn as dots for patient MA in No Vision, Alternate No Vision, and Alternate Arm Vision conditions, small filled circles show target locations. $B$ : individual hand paths (plotted as in Fig. 1) and velocity profiles of 2 movements to show how End1 and End 2 are marked. $C$ : mean distance $\pm \mathrm{SE}$ between End 1 and End 2 for 2 patients $(M A$ and $C F)$ in No Vision (shaded bars), Alternate No Vision (hatched bars), and Alternate Arm Vision (unfilled bars).

used to form and/or update an internal representation, or model, of the limb. Because the improvements seemed more substantial during movements made after viewing the arm in motion (on alternate trials) than with vision of the limb at rest (which in patient $C F$ were negligible), information obtained in peripheral vision during limb motion may be important in updating dynamic parameters.

What information did prior vision provide and how was it used in improving the programming of movements carried out without feedback? Vision of the limb at rest provides information about configuration of the limb (e.g., joint angles and segment lengths), its relationship to the body, and its location relative to nearby objects and surfaces. Monitoring the arm or the motion of the screen cursor during movement provides additional information about the dynamic properties of the limb and its response to immediately preceding neural commands. In the case of the cursor display, this includes information about the effects of corrections instituted in the course of the trajectory. Such information is likely to be especially critical for calibrating both proprioceptive and internal feedback signals as well as for interpreting trajectory errors. It thus may be critical in adaptive adjustments of motor programs governing subsequent movements. It should be noted, however, that subjects may be able to infer some dynamic characteristics of the limb, such as inertial anisotropy, by viewing it at rest. Indeed, when subjects view external objects that they intend to grasp, they make inferences about such object properties as weight, friction, and other parameters (Johansson and Cole 1992) in programming movement. Therefore, the reduction of movement errors made after having viewed the screen cursor alone (rather than the arm), provides the strongest evidence for the importance of prior information about limb dynamics in movement programming.

It is interesting to note that because the path of the cursor was always displayed after each movement, trials with and without feedback differed primarily in the time at which this information was made available. As was the case in trials with vision of the limb, cursor feedback allowed subjects to compare, concurrently, the evolving movement with the expected trajectory. We have found that cursor feedback is also more effective than $\mathrm{KR}$ in enabling normal controls to compensate for directional biases that depend on the initial position of the hand (Ghez et al. 1994; Ghilardi et al. 1994). 


\section{Patient M.A.}

\section{A Movement Paths}

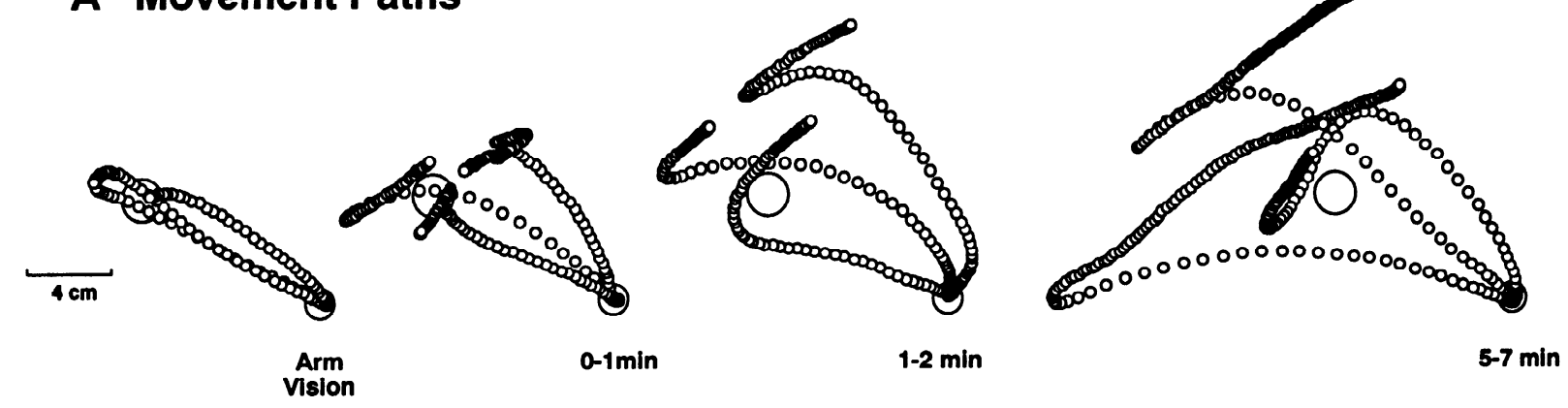

B Mean Movement Error \pm S.E.

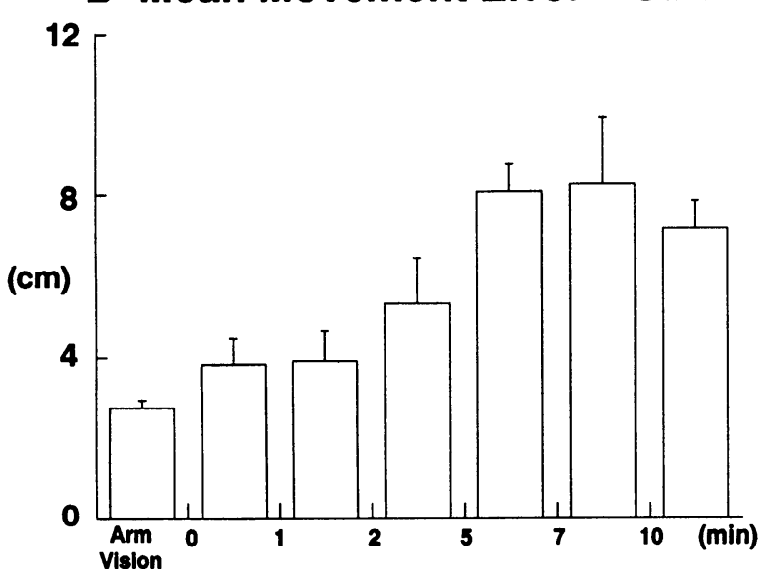

C Mean Secondary Movement \pm S.E.

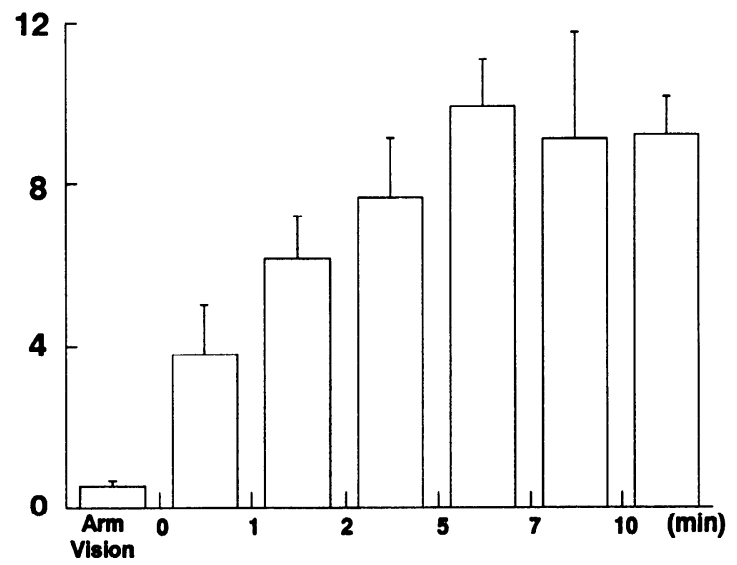

FIG. 7. Decay of the effect of limb vision as function of time in patient MA. Data averaged across 2 similar blocks of Arm Vision followed by No Vision trials (see text). A: hand paths shown as small circles drawn at 20-ms intervals. $B:$ mean error of primary movement (distance of target to End1) \pm SE. $C$ : mean secondary movement extents \pm SE in trials with arm vision and without arm vision. The moment when the arm was covered is time 0 . Other numbers refers to the time in minutes after that moment. Number of trials comprising time intervals: arm vision, $48 ; 0-1 \mathrm{~min}, 8 ; 1-2 \mathrm{~min}, 19 ; 2-5 \mathrm{~min}$, 14 ; 5-7 $\mathrm{min}, 19 ; 7-10 \mathrm{~min}, 7$; and $\geq 10 \mathrm{~min}, 26$.

\section{Compensation for direction-dependent variations in limb inertia}

A characteristic feature of the movements made by deafferented patients is their dependence on limb inertia. Both control subjects and patients show marked anisotropy in initial hand acceleration reflecting direction-dependent differences in inertia. Control subjects compensate for this by systematically varying movement time, but the mechanisms underlying this compensation are not clear. We have discussed three possible mechanisms that could account for this in normal subjects (Gordon et al. 1994a). The question therefore arises whether the same mechanisms could enable the compensation observed in deafferented patients when they use vision.

One possibility is that in normals, this compensation is mediated by muscle spindle feedback to motoneurons, which under gamma drive could signal a mismatch between an intended and desired position of the limb [e.g., as in the servo-assistance hypothesis proposed by Matthews (1972), and the lambda model of Feldman et al. (1990)]. The failure to adjust movement time in patients would appear to favor this hypothesis because muscle spindle input is abolished by peripheral neuropathy. However, if proprioceptively mediated reflex mechanisms were to be necessary for this compensation, then visual information from previous movements should be without effect. Thus our finding that prior vision of the limb allows deafferented patients to better adapt movement time to direction indicates that such proprioceptive reflexes are not essential for inertial compensation.

A second possibility is that movement time is controlled by feedforward mechanisms - presumably based on an internal model of limb mechanical properties - to counter expected variations in inertia. Updating of internal models of limb through vision could explain the improved control over movement time in patients without proprioception. An objection is that if it could compute inertia, the nervous system also should be able to match the initial force to the inertia of the limb (Gordon et al. 1994a). Nevertheless, such a mechanism might be available to both controls and patients, and, in the absence of proprioception, the patients might rely on this to a greater degree than controls.

A third possibility is that compensation results from lags 
introduced by passive viscoelastic properties of muscles and joints as a new equilibrium position is being specified (also through feedforward mechanisms) by descending commands. The question therefore arises as to how patients successfully compensate for variations in limb inertia with prior vision and whether the deafferented patients do so in the same way as do subjects with intact proprioception. One possibility is that the nervous system varies movement time by varying the timing of muscle commands, based, for example, on an internal model of limb inertia. Alternatively, the direction-dependent variations in movement time in control subjects might emerge as a consequence of the interactions of limb inertia and joint stiffness associated with changes in equilibrium position (Bizzi et al. 1984; Flash 1990; Hogan 1988).

One version of this idea, Feldman's lambda model, proposes that the regulation of joint stiffness depends on proprioceptive feedback. This idea cannot, as noted earlier, readily account the effect of vision in deafferented patients. The improved trajectory control in deafferented patients produced by vision does not necessarily contradict the view that proprioceptive feedback is critical for normal joint compliance (Houk and Rymer 1981). Rather it suggests that impairments in these low-level control mechanisms can, at least to some extent, be compensated by descending controls. For example, in the absence of proprioception, joint stiffness might be regulated more directly by increasing descending drive to antagonist muscles.

Another aspect of Feldman's suggestion (1990) may be more relevant, namely that subjects program movements via a reciprocally organized " $R$-command" and a cocontractive "C-command". The former would determine a spatial trajectory whereas the latter would increase joint stiffness during movement and maintain posture at the end of movement. The presence of secondary movements that, in patients, take the hand in different directions suggest that distinct processes specify the transport phase and the terminal end point. The different times at which these commands are implemented implies different time courses and, potentially, regulation by different mechanisms. This possibility is in accord with the observation of Humphrey and Reed (1983) that different populations of motor cortex neurons become active during reciprocal activation and cocontraction of antagonists. It also fits with the recent finding that the electromyograms of upper extremity muscles during reaching movements can, by principal components analysis, be approximated by the summed contributions of independent tonic and phasic components (Flanders and Herrmann 1992).

The inaccuracy of movements made by deafferented patients without vision then could result from the combined effects of errors in specifying the intended direction and magnitude of an initial force pulse and errors in specifying a terminal end point. Mismatches between the intended posture at the end of movement and the spatial location toward which the hand was aimed could produce the bends between the primary transport phase and the late secondary movements. By updating the subjects' internal limb model, vision of the limb would improve the planning of both the phasic and tonic commands. Our finding that prior vision dramati- cally reduces terminal drifts in the patients would fit with this hypothesis.

\section{Importance of internal models of the limb for motor learning}

The marked improvement in performance produced by vision of the limb on preceding trials raises the question of how long this effect persists. Our results indicate that performance is considerably degraded within a few minutes once vision of the limb is no longer available. It may appear surprising that the effects of vision of the limb in patients and therefore the representation of the limb's properties in memory, should be so transitory. It suggests that internal models of the dynamic properties of the limb used for planning reaching movements require continuous updating. Such plasticity of internal models might represent an adaptation to the susceptibility of muscles to changes in mechanical properties (e.g., with fatigue, changes in temperature) and to the possibility of varied mechanical loads (e.g., tool use, orientation relative to gravity). It recently has been shown that during catching behaviors there are substantial and complex changes in limb impedance control (Lacquaniti et al. 1992,1993 ). Without a mechanism for rapidly updating the representation of limb mechanical properties, such changes would impair accuracy in complex multijoint tasks. Similar roles for internal models of the limb, body, and the environment have been proposed for other forms of limb control (Atkeson 1989; Jordan and Rumelhart 1992; Lacquaniti et al. 1992, 1993; Morasso and Sanguineti 1992) and for the control of posture (Gurfinkel et al. 1988). Our observations therefore provide support for computational models of movement planning and control that postulate the existence and importance of internal models of the dynamic properties of the limb.

\begin{abstract}
We are very grateful to Drs. Yves Lamarre and Robert Forget for allowing us to study patient $G L$ and to Dr. Oliver Sacks for introducing us to patient $C F$. We also are indebted to Drs. Roberto Bermejo and Meenakshi Iyer who provided invaluable assistance to us during some experiments in this series. This work was supported by National Institute of Neurological Disorders and Stroke Grant NS-22715 and the McKnight Foundation.

Address for reprint requests: $\mathrm{C}$. Ghez, Center for Neurobiology and Behavior, 722 West 168th St., New York, NY 10032.
\end{abstract}

Received 14 April 1994; accepted in final form 8 September 1994.

\section{REFERENCES}

Adams, R. D. AND Victor, M. Principles of Neurology (2nd ed.). New York: McGraw-Hill, 1981.

AtKeson, C. G. Learning arm kinematics and dynamics. Annu. Rev. Neurosci. 12: 157-183, 1989.

Bizzi, E., Accornero, N., Chapple, W., AND Hogan, N. Posture control and trajectory formation during arm movement. J. Neurosci. 4: 2738 2744, 1984.

Cleveland, W. S. Robust locally weighted regression and smoothing scatterplots. J. Am. Stat. Assn. 74: 829-836, 1979.

Feldman, A. G., Adamovich, S. V., Ostry, D. J., ANd Flanagan, J. R. The origin of electromyograms-Explanations based on the equilibrium point hypothesis. In: Multiple Muscle Systems: Biomechanics and Movement Organization, edited by J. M. Winters and S. L.-Y. Woo. New York: Springer-Verlag, 1990, p. 195-213.

FLANDERS, M., AND HERRMANN, U. Two components of muscle activation: scaling with the speed of arm movement. J. Neurophysiol. 6?: 931-943, 1992. 
FLASH, T. The organization of human arm trajectory control. In: Multiple Muscle Systems: Biomechanics and Movement Organization, edited by J. M. Winters and S. L.-Y. Woo. New York: Springer-Verlag, 1990, p. 282-301.

FORGET, R. AND LAMARRE, Y. Rapid elbow flexion in the absence of proprioceptive and cutaneous feedback. Hum. Neurobiol. 6: 27-37, 1987.

GHEz, C. The control of movement. In: Principles of Neural Science, edited by E. Kandel, J. Schwartz, and T. Jessell. New York: Elsevier, 1991, p. 533-547.

GHEZ, C., BERMEJO, R., AND GoRDON, J. Impairment in programming of response direction and amplitude in deafferented patients. Soc. Neurosci. Abstr. 14: 953, 1988.

Ghez, C., Gordon, J., Ghilardi, M. F., Christakos, C. N., AND CoOPER, $S$. E. Roles of proprioceptive input in the programming of arm trajectories. Cold Spring Harbor Symp. Quant. Biol. 55: 837-847, 1990.

Ghez, C., Gordon, J., Ghilardi, M. F., and SaInBurg, R. Contributions of vision and proprioception to accuracy in limb movements. In: The Cognitive Neurosciences, edited by M. S. Gazzaniga. Cambridge, MA: MIT Press, 1994, p. 549-564.

GHILARDI, M. F., Gordon, J., AND GHEZ, C. Directional biases in arm movements reflect biased estimates of initial hand position. Soc. Neurosci. Abstr. 20: 1410, 1994.

Gordon, J., Ghilardi, M. F., CoOPER, S. E., ANd Ghez, C. Accuracy of planar reaching movements. II. Systematic extent errors resulting from inertial anisotropy. Exp. Brain Res. 99: 112-130, 1994a.

Gordon, J., GHILARDI, M. F., AND GHEZ, C. Deafferented subjects fail to compensate for workspace anisotropies in 2-dimensional arm movements. Soc. Neurosci. Abstr. 16: 1089, 1990.

Gordon, J., GhILARDI, M. F., AND GHEz, C. Accuracy of planar reaching movements I. Independence of direction and extent variability. Exp. Brain Res. 99: 97-111, 1994b.

Gordon, J., GHILARDI, M. F., AND GHEZ, C. Impairments of reaching movements in patients without proprioception. I. Spatial errors. J. Neurophysiol. 73: 347-360, 1995.

GORDON, J., IYER, M., AND GHEZ, C. Impairment of motor programming and trajectory control in a deafferented patient. Soc. Neurosci. Abstr. 13. $352,1987$.

Gurfinkel, V. S., Levick, Y. S., Popov, K. E., SMetanin, B. N., AND SHLIKOV, V. Y. Body scheme in the control of postural activity. In:
Stance and Motion: Facts and Concepts, edited by V. S. I. Gurfinkel, M. E. J. Massion, and J. P. Roll. New York: Plenum, 1988, p. 185-193. Hogan, N. Planning and execution of multijoint movements. Can. J. Physiol. Pharmacol. 66: 508-517, 1988.

HouK, J. C. AND RYMER, W. Z. Neural control of muscle length and tension. In: Handbook of Physiology. The Nervous System. Bethesda, MD: Am. Physiol. Soc., 1981, sect. 1. vol. II, part 1, p. 257-323.

HuMPHREY, D. R. AND REED, D. J. Separate cortical systems for control of joint movement and joint stiffness: reciprocal activation and coactivation of antagonist muscles. In: Advances in Neurology, Motor Control Mechanisms in Health and Disease, edited by J. E. Desmedt. New York: Raven Press, 1983, vol. 39, p. 347-372.

Johansson, R. S. AND Cole, K. J. Sensory-motor coordination during grasping and manipulative actions. Curr. Opin. Neurobiol. 2: 815-823, 1992.

JORDAN, M. I. AND RUMELHART, D. E. Forward models: supervised learning with a distal teacher. Cognit. Sci. 16: 307-354, 1992.

LACQUANiti, F., BorgheSE, N. A., AND CARrozzo, M. Internal models of limb geometry in the control of hand compliance. J. Neurosci. 12: 1750$1762,1992$.

Lacquaniti, F., Carrozzo, M., and Borghese, N. A. Time-varying mechanical behavior of multijointed arm in man. J. Neurophysiol. 69: 14431464, 1993.

Matrhews, P. B. C. Mammalian Muscle Receptors and Their central Actions. London: Arnold, 1972

MoRASSO, P. AND SANGUINETI, V. Equilibrium point and self-organization. Behav. Brain Sci. 15: 781-782, 1992.

NiCHOLS, T. R. AND Houk, J. C. Improvement in linearity and regulation of stiffness that results from actions of stretch reflex. J. Neurophysiol. 39: $119-142,1976$.

Rothwell, J. L., Traub, M. M., Day, B. L., Obeso, J. A., Thomas, P. K., AND MARSDEN, C. D. Manual motor performance in a deafferented man. Brain 105: 515-542, 1982.

Sainburg, R. L., GHILARDI, M. F., PoIzNER, H., AND GHez, C. Vision of the limb allows deafferented patients to control multijoint dynamics. Soc. Neurosci. Abstr. 19: 1686, 1993.

Sanes, J. N., Mauritz, K.-H., DalaKas, M. C., and EvarTs, E. V. Motor control in humans with large-fiber sensory neuropathy. Hum. Neurobiol. 4: $101-114,1985$ 